\title{
The enclosure method for the heat equation
}

\author{
Masaru IKEHATA \\ Department of Mathematics, Graduate School of Engineering \\ Gunma University, Kiryu 376-8515, JAPAN \\ Mishio KAWASHITA \\ Department of Mathematics, Graduate School of Sciences \\ Hiroshima University, Higashi Hiroshima 739-8526, JAPAN \\ final
}

\begin{abstract}
This paper shows how the enclosure method which was originally introduced for elliptic equations can be applied to inverse initial boundary value problems for parabolic equations. For the purpose a prototype of inverse initial boundary value problems whose governing equation is the heat equation is considered. An explicit method to extract an approximation of the value of the support function at a given direction of unknown discontinuity embedded in a heat conductive body from the temperature for a suitable heat flux on the lateral boundary for a fixed observation time is given.

AMS: 35R30, 80A23

KEY WORDS: inverse initial boundary value problem, heat equation, parabolic equation, thermal imaging, cavity, corrosion, enclosure method
\end{abstract}

\section{Introduction}

The aim of this paper is to show how the enclosure method which was originally introduced for elliptic equations in [4] can be applied to inverse initial boundary value problems for parabolic equations in multi-dimensions. We present only a simple case just for the explanation of the idea, however, the same idea will work also for more general cases.

Let $\Omega$ be a bounded domain of $\mathbf{R}^{m}, m=2,3$ with a smooth boundary. Let $D$ be an open subset of $\Omega$ with a smooth boundary and satisfy that: $\bar{D} \subset \Omega ; \Omega \backslash \bar{D}$ is connected. We denote the unit outward normal vectors to $\partial \Omega$ and $\partial D$ by the same symbol $\nu$. Let $T$ be an arbitrary fixed positive number.

Given $f=f(x, t),(x, t) \in \partial \Omega \times] 0, T[$ let $u=u(x, t)$ be the solution of the initial 
boundary value problem for the heat equation:

$$
\begin{gathered}
\left.\partial_{t} u-\triangle u=0 \text { in }(\Omega \backslash \bar{D}) \times\right] 0, T[, \\
\left.\frac{\partial u}{\partial \nu}=0 \text { on } \partial D \times\right] 0, T[, \\
\left.\frac{\partial u}{\partial \nu}=f \text { on } \partial \Omega \times\right] 0, T[, \\
u(x, 0)=0 \text { in } \Omega \backslash \bar{D} .
\end{gathered}
$$

This paper considers the following problem for an explanation of the idea.

Inverse Problem. Assume that $D$ is unknown. Extract information about the location and shape of $D$ from the temperature $u(x, t)$ and heat flux $f(x, t)$ for $(x, t) \in \partial \Omega \times] 0, T[$.

The set $D$ is a model of the union of unknown cavities or subregions where the heat conductivity is very low compared with that of the surrounded region $\Omega \backslash \bar{D}$. Thus the problem is a mathematical formulation of a typical inverse problem in thermal imaging. The method to solve this inverse problem may have possibility of application in nondestructive evaluation.

There are extensive studies on the uniqueness and stability issues on this kind of problems. See [10] and references therein for the issues. In this paper we are concerned with the reconstruction issue. Note that in [6] Ikehata studied this type of problems in one-space dimensional case by using the enclosure method. In [7] he raised several questions related to the method in multi-dimensional cases. However, it is still not clear that the method works also for two or three-space dimensional cases since the study in [6] fully makes use of the speciality of one-space dimension.

Our main result is the following formula.

Theorem 1.1. Given $\omega \in S^{m-1}$ let $f$ be the function of $\left.(x, t) \in \partial \Omega \times\right] 0, T[$ having a parameter $\tau>0$ defined by the equation

$$
f(x, t ; \tau)=\frac{\partial v}{\partial \nu}(x) \varphi(t),
$$

where $v(x)=e^{\sqrt{\tau} x \cdot \omega}$ and $\varphi \in L^{2}(0, T)$ satisfying the condition: there exists $\mu \in \mathbf{R}$ such that

$$
\liminf _{\tau \longrightarrow \infty} \tau^{\mu}\left|\int_{0}^{T} e^{-\tau t} \varphi(t) d t\right|>0 .
$$

Let $u_{f}=u_{f}(x, t)$ be the weak solution of (1.1) for $f=f(x, t ; \tau)$ and $h_{D}(\omega)=\sup _{x \in D} x \cdot \omega$. Then the formula

$$
\lim _{\tau \longrightarrow \infty} \frac{1}{2 \sqrt{\tau}} \log \left|\int_{\partial \Omega} \int_{0}^{T} e^{-\tau t}\left(v(x) f(x, t ; \tau)-u_{f}(x, t) \frac{\partial v}{\partial \nu}(x)\right) d t d S\right|=h_{D}(\omega),
$$

is valid.

Note that: if $\varphi(t)$ is smooth on $[0, \delta[$ with $0<\delta<T$ and $t=0$ is not a zero point with infinite order of $\varphi(t)$, then (1.3) is satisfied for an appropriate $\mu>0$. 
The function $h_{D}(\cdot)$ is called the support function of $D$ which gives the signed distances from the origin of coordinates to the support plane $(m=3)$, line $(m=2)$ of $D$. Since the convex hull of $D$ is given by the formula $\cap_{\omega \in S^{m-1}}\left\{x \in \mathbf{R}^{m} \mid x \cdot \omega<h_{D}(\omega)\right\}$, using the formula (1.4), we obtain the set $\left\{x \in \mathbf{R}^{m} \mid x \cdot \omega<h_{D}(\omega)\right\}$ which gives an upper bound of the convex hull of $D$ from the direction $\omega$.

In [6] Ikehata considered the corresponding problem in one-space dimension for the equation $\partial_{t} u=\left(\gamma u_{x}\right)_{x}$ with a smooth $\gamma$. Theorem 1.1 can be considered as an extension of (2) of Theorem 2.5 in [6] in the case $\gamma=1$ to two and three-space dimensional cases. Note that therein the parameter $\tau^{2}$ plays a role of $\tau$ in Theorem 1.1.

A brief outline of this paper is as follows. Theorem 1.1 is proved in Subsection 2.2. The proof is based on an integral identity which is described in Subsubsection 2.2.1. Using the identity, we give an asymptotic representation formula of the integral

$$
\int_{\partial \Omega} \int_{0}^{T} e^{-\tau t}\left(v(x) f(x, t ; \tau)-u_{f}(x, t) \frac{\partial v}{\partial \nu}(x)\right) d t d S
$$

together with an estimate of the leading term which is an integral involving two Neumannto-Dirichlet maps for the operator $\triangle-\tau$. The estimate of the remainder term is described in Subsubsection 2.2.2 as Lemma 2.1 and that of the leading term is found in the same subsubsection as Lemma 2.2. To establish Lemma 2.1 we require an energy estimate of $u_{f}(\cdot, T)$ : this together with the meaning of the weak solution of (1.1) is found in Subsection 2.1. In Section 3 we show how Theorem 1.1 can be extended also to extract another information about $D$, that is the distance from a given $p$ outside $\Omega$ to $D$.

\section{The enclosure method}

In this section firstly we specify what we mean by the weak solution of (1.1) and describe a necessary estimate. Secondly by giving the proof of Theorem 1.1 we show how the enclosure method can be applied to the heat equation.

\subsection{Preliminaries about the direct problem.}

We follow [1]. For two separable Hilbert spaces $V$ and $H$ with $V \subset H \subset V^{\prime}$, and a positive number $T$, the space $W\left(0, T ; V, V^{\prime}\right)$ is defined by $W\left(0, T ; V, V^{\prime}\right)=\{u \mid u \in$ $\left.L^{2}(0, T ; V), u^{\prime} \in L^{2}\left(0, T ; V^{\prime}\right)\right\}$. Note that $u^{\prime}$ means the derivative in $\left.t \in\right] 0, T[$.

Given $f \in L^{2}\left(0, T ; H^{-1 / 2}(\partial \Omega)\right)$ we say that $u \in W\left(0, T ; H^{1}(\Omega \backslash \bar{D}),\left(H^{1}(\Omega \backslash \bar{D})\right)^{\prime}\right)$ satisfy

$$
\begin{aligned}
\partial_{t} u-\triangle u & =0 \text { in }(\Omega \backslash \bar{D}) \times] 0, T[, \\
\frac{\partial u}{\partial \nu} & =0 \text { on } \partial D \times] 0, T[, \\
\frac{\partial u}{\partial \nu} & =f \text { on } \partial \Omega \times] 0, T[
\end{aligned}
$$

in the weak sense if the $u$ satisfies

$$
\frac{d}{d t} \int_{\Omega \backslash \bar{D}} u(x, t) \varphi(x) d x+\int_{\Omega \backslash \bar{D}} \nabla u(x, t) \cdot \nabla \varphi(x) d x=<f(t),\left.\varphi\right|_{\partial \Omega}>\operatorname{in}(0, T),
$$


in the sense of distribution on $(0, T)$ for all $\varphi \in H^{1}(\Omega \backslash \bar{D})$. By (1.50) on page 477 in [1] we have

$$
\frac{d}{d t} \int_{\Omega \backslash \bar{D}} u(x, t) \varphi(x) d x=<u^{\prime}(t), \varphi>
$$

in the sense of distribution on $(0, T)$ for all $\varphi \in H^{1}(\Omega \backslash \bar{D})$. Thus (2.2) is valid also for $t \in] 0, T[$ a.e..

Note that by Theorem 1 on p.473 in [1] we see that every $u \in W\left(0, T ; H^{1}(\Omega \backslash\right.$ $\left.\bar{D}),\left(H^{1}(\Omega \backslash \bar{D})\right)^{\prime}\right)$ is almost everywhere equal to a continuous function of $[0, T]$ in $L^{2}(\Omega \backslash \bar{D})$. Further, we have:

$$
W\left(0, T ; H^{1}(\Omega \backslash \bar{D}),\left(H^{1}(\Omega \backslash \bar{D})\right)^{\prime}\right) \hookrightarrow C^{0}\left([0, T] ; L^{2}(\Omega \backslash \bar{D})\right),
$$

the space $C^{0}\left([0, T] ; L^{2}(\Omega \backslash \bar{D})\right)$ being equipped with the norm of uniform convergence. Thus one can consider $u(0)$ and $u(T)$ as elements of $L^{2}(\Omega \backslash \bar{D})$. Then by Theorems 1 and 2 on p.512 and 513 in [1] we see that given $u_{0} \in L^{2}(\Omega \backslash \bar{D})$ there exists a unique $u$ such that $u$ satisfies (2.1) in the weak sense and satisfies the initial condition $u(0)=u_{0}$.

Let $u_{0}=0$. By Remark 2 on p.512 and Theorem 3 on p.520 in [1] we have the continuity of $u$ on $f$ : there exists a $C_{T}>0$ independent of $f$ such that

$$
\|u\|_{L^{2}\left(0, T ; H^{1}(\Omega \backslash \bar{D})\right)} \leq C_{T}\|f\|_{L^{2}\left(0, T ; H^{-1 / 2}(\partial \Omega)\right)} .
$$

Moreover, from (2.2) and (2.4) we have

$$
\left\|u^{\prime}\right\|_{L^{2}\left(0, T ; H^{1}(\Omega \backslash \bar{D})^{\prime}\right)} \leq C_{T}\|f\|_{L^{2}\left(0, T ; H^{-1 / 2}(\partial \Omega)\right)} .
$$

This together with (2.3) and (2.4) yields one of the important estimates in the enclosure method:

$$
\|u(T)\|_{L^{2}(\Omega \backslash \bar{D})} \leq C_{T}\|f\|_{L^{2}\left(0, T ; H^{-1 / 2}(\partial \Omega)\right)} .
$$

In the following subsection we denote by $u_{f}$ the weak solution of $(2.1)$ with $u(0)=0$ and this is the meaning of the weak solution of (1.1).

\subsection{Proof of Theorem 1.1}

Define

$$
w_{f}(x ; \tau)=\int_{0}^{T} e^{-\tau t} u_{f}(x, t) d t, x \in \Omega \backslash \bar{D}
$$

and

$$
g_{f}(x ; \tau)=\int_{0}^{T} e^{-\tau t} f(x, t) d t, x \in \partial \Omega,
$$

where $\tau>0$ is a parameter. This type of transform has been used in the study [6] for the corresponding problem in a one-space dimensional case. 


\subsubsection{The basic identity}

The function $w_{f}$ satisfies

$$
\begin{gathered}
(\triangle-\tau) w_{f}=e^{-\tau T} u_{f}(x, T) \text { in } \Omega \backslash \bar{D} \\
\frac{\partial w_{f}}{\partial \nu}=0 \text { on } \partial D \\
\frac{\partial w_{f}}{\partial \nu}=g_{f} \text { on } \partial \Omega
\end{gathered}
$$

Let $v=v(x)$ satisfy $(\triangle-\tau) v=0$ in $\Omega$. Integration by parts yields

$$
\int_{\partial \Omega}\left(g_{f} v-w_{f} \frac{\partial v}{\partial \nu}\right) d S=-\int_{\partial D} w_{f} \frac{\partial v}{\partial \nu} d S+e^{-\tau T} \int_{\Omega \backslash \bar{D}} u_{f}(x, T) v(x) d x .
$$

Let $p_{f}$ be the unique solution of the boundary value problem:

$$
\begin{gathered}
(\triangle-\tau) p=0 \text { in } \Omega \backslash \bar{D} \\
\frac{\partial p}{\partial \nu}=0 \text { on } \partial D \\
\frac{\partial p}{\partial \nu}=g_{f} \text { on } \partial \Omega
\end{gathered}
$$

Set $\epsilon_{f}=w_{f}-p_{f}$. Since we have

$$
\int_{\partial \Omega}\left(g_{f} v-p_{f} \frac{\partial v}{\partial \nu}\right) d S=-\int_{\partial D} p_{f} \frac{\partial v}{\partial \nu} d S
$$

from (2.6) we obtain the basic identity:

$$
\begin{gathered}
\int_{\partial \Omega}\left(g_{f} v-w_{f} \frac{\partial v}{\partial \nu}\right) d S \\
=\int_{\partial \Omega}\left(g_{f} v-p_{f} \frac{\partial v}{\partial \nu}\right) d S-\int_{\partial D} \epsilon_{f} \frac{\partial v}{\partial \nu} d S+e^{-\tau T} \int_{\Omega \backslash \bar{D}} u_{f}(x, T) v(x) d x .
\end{gathered}
$$

Note that $\epsilon_{f}$ satisfies

$$
\begin{gathered}
(\triangle-\tau) \epsilon_{f}=e^{-\tau T} u_{f}(x, T) \text { in } \Omega \backslash \bar{D}, \\
\frac{\partial \epsilon_{f}}{\partial \nu}=0 \text { on } \partial D \\
\frac{\partial \epsilon_{f}}{\partial \nu}=0 \text { on } \partial \Omega
\end{gathered}
$$

Multiplying the first equation in (2.8) by $\epsilon_{f}$ and integrating over $\Omega \backslash \bar{D}$, we have

$$
\int_{\Omega \backslash \bar{D}}\left|\nabla \epsilon_{f}\right|^{2} d x+\tau \int_{\Omega \backslash \bar{D}}\left|\epsilon_{f}\right|^{2} d x=-e^{-\tau T} \int_{\Omega \backslash \bar{D}} u_{f}(x, T) \epsilon_{f}(x) d x .
$$

This right hand side has the bound $e^{-\tau T}\left\|u_{f}(T)\right\|_{L^{2}(\Omega \backslash \bar{D})}\left\|\epsilon_{f}\right\|_{L^{2}(\Omega \backslash \bar{D})}$. Since $\tau>0$, we have immediately

$$
\left\|\epsilon_{f}\right\|_{H^{1}(\Omega \backslash \bar{D})} \leq\left(\tau^{-1}+\tau^{-2}\right)^{1 / 2} e^{-\tau T}\left\|u_{f}(T)\right\|_{L^{2}(\Omega \backslash \bar{D})} .
$$




\subsubsection{Two lemmas}

Now we choose a special $f$ having the form:

$$
\left.f(x, t ; \tau)=\frac{\partial v}{\partial \nu}(x) \varphi(t),(x, t) \in \partial \Omega \times\right] 0 T[
$$

where $\varphi \in L^{2}(0, T)$. For this $f$ we have

$$
\|f\|_{L^{2}\left(0, T ; H^{-1 / 2}(\partial \Omega)\right)}=\|\varphi\|_{L^{2}(0, T)}\left\|\frac{\partial v}{\partial \nu}\right\|_{H^{-1 / 2}(\partial \Omega)} .
$$

Since

$$
g_{f}(x ; \tau)=\frac{\partial v}{\partial \nu}(x) \int_{0}^{T} e^{-\tau t} \varphi(t) d t
$$

we have the expression of the first term of the right hand side of (2.7):

$$
\int_{\partial \Omega}\left(g_{f} v-p_{f} \frac{\partial v}{\partial \nu}\right) d S=\int_{0}^{T} e^{-\tau t} \varphi(t) d t \int_{\partial \Omega} \frac{\partial v}{\partial \nu}\left(R_{\emptyset}(\tau)-R_{D}(\tau)\right) \frac{\partial v}{\partial \nu} d S
$$

where $R_{\emptyset}(\tau)$ and $R_{D}(\tau)$ are the Neumann-to-Dirichlet maps on $\partial \Omega$ for the operator $\triangle-\tau$ in $\Omega$ and $\Omega \backslash \bar{D}$ with the homogeneous Neumann boundary condition on $\partial D$, respectively.

Hereafter we choose a special $v$ in $(2.10)$ which is a solution of the equation $(\triangle-\tau) v=0$ in $\mathbf{R}^{m}$ :

$$
v(x)=e^{\sqrt{\tau} x \cdot \omega}
$$

where $\omega \in S^{m-1}$ and $\tau>0$. Thus, we choose $f(x, t ; \tau)$ as described in (1.2).

The following is an easy consequence of the estimates $(2.5),(2.9)$ and the growth order of $v$ as $\tau \longrightarrow \infty$.

Lemma 2.1. We have, as $\tau \longrightarrow \infty$

$$
-\int_{\partial D} \epsilon_{f} \frac{\partial v}{\partial \nu} d S+e^{-\tau T} \int_{\Omega \backslash \bar{D}} u_{f}(x, T) v(x) d x=O\left(\tau^{\gamma} e^{-\tau T} e^{2 \sqrt{\tau} h_{\Omega}(\omega)}\right)
$$

where $\gamma$ is a positive constant and $h_{\Omega}(\omega)=\sup _{x \in \Omega} x \cdot \omega$.

Proof. First we show that the first term in (2.13) has the bound $O\left(\tau^{3 / 2} e^{-\tau T} e^{2 \sqrt{\tau} h_{\Omega}(\omega)}\right)$ as $\tau \longrightarrow \infty$. Since $v$ satisfies $(\triangle-\tau) v=0$ in $\Omega$, we have, for all $\eta \in H^{1}(\Omega)$

$$
\int_{\partial \Omega} \frac{\partial v}{\partial \nu} \eta d S=\int_{\Omega} \nabla v \cdot \nabla \eta d x+\tau \int_{\Omega} v \eta d x
$$

This gives

$$
\left|\int_{\partial \Omega} \frac{\partial v}{\partial \nu} \eta d S\right| \leq\left(\|\nabla v\|_{L^{2}(\Omega)}+\tau\|v\|_{L^{2}(\Omega)}\right)\|\eta\|_{H^{1}(\Omega)} .
$$

Since $\eta$ is arbitrary and the trace operator $H^{1}(\Omega) \longrightarrow H^{1 / 2}(\partial \Omega)$ has a bounded right inverse, we have

$$
\left\|\frac{\partial v}{\partial \nu}\right\|_{H^{-1 / 2}(\partial \Omega)} \leq C\left(\|\nabla v\|_{L^{2}(\Omega)}+\tau\|v\|_{L^{2}(\Omega)}\right)
$$


Similarly we have

$$
\left\|\frac{\partial v}{\partial \nu}\right\|_{H^{-1 / 2}(\partial D)} \leq C\left(\|\nabla v\|_{L^{2}(D)}+\tau\|v\|_{L^{2}(D)}\right) .
$$

Since the trace operator $H^{1}(\Omega \backslash \bar{D}) \longrightarrow H^{1 / 2}(\partial D)$ is bounded, we have

$$
\left|\int_{\partial D} \epsilon_{f} \frac{\partial v}{\partial \nu} d S\right| \leq C\left\|\frac{\partial v}{\partial \nu}\right\|_{H^{-1 / 2}(\partial D)}\left\|\epsilon_{f}\right\|_{H^{1}(\Omega \backslash \bar{D})}
$$

Now from this, (2.9) and (2.15) we obtain

$$
\left|\int_{\partial D} \epsilon_{f} \frac{\partial v}{\partial \nu} d S\right| \leq C\left(\tau^{-1}+\tau^{-2}\right)^{1 / 2}\left(\|\nabla v\|_{L^{2}(\Omega)}+\tau\|v\|_{L^{2}(\Omega)}\right) e^{-\tau T}\left\|u_{f}(T)\right\|_{L^{2}(\Omega \backslash \bar{D})} .
$$

This together with the special form of $v,(2.5),(2.11)$ and (2.14) gives the desired estimate.

It is clear that the second term of (2.13) has the bound $O\left(\tau e^{-\tau T} e^{2 \sqrt{\tau} h_{\Omega}(\omega)}\right)$ as $\tau \longrightarrow \infty$.

The integral in (1.4) is just the left-hand side of (2.7). This fact, (2.12) and Lemma 2.1 imply that

$$
\begin{gathered}
\int_{\partial \Omega} \int_{0}^{T} e^{-\tau t}\left(v(x) f(x, t ; \tau)-u_{f}(x, t) \frac{\partial v}{\partial \nu}(x)\right) d t d S \\
=\int_{0}^{T} e^{-\tau t} \varphi(t) d t \int_{\partial \Omega} \frac{\partial v}{\partial \nu}\left(R_{\emptyset}(\tau)-R_{D}(\tau)\right) \frac{\partial v}{\partial \nu} d S+O\left(\tau^{\gamma} e^{-\tau T} e^{2 \sqrt{\tau} h_{\Omega}(\omega)}\right) .
\end{gathered}
$$

Thus, the main contribution on the limit (1.4) is the term given by two Neumann-toDirichlet maps. For this term we need the following estimates from the both sides:

Lemma 2.2. There exist $\mu_{1}, \mu_{2} \in \mathbf{R}, \tau_{0}>0, C_{1}>0$ and $C_{2}>0$ such that, for all $\tau>\tau_{0}$

$$
C_{1} \tau^{\mu_{1}} \leq e^{-2 \sqrt{\tau} h_{D}(\omega)}\left|\int_{\partial \Omega} \frac{\partial v}{\partial \nu}\left(R_{\emptyset}(\tau)-R_{D}(\tau)\right) \frac{\partial v}{\partial \nu} d S\right| \leq C_{2} \tau^{\mu_{2}}
$$

Note that in Lemma 2.2 the lower estimate is essential and the strict values of $\mu_{1}$ and $\mu_{2}$ are not important to obtain formula (1.4).

From Lemma 2.2, (2.16) and (1.3), we have formula (1.4) in Theorem 1.1. More precisely, the condition (1.3) means that there exist positive constants $C$ and $\tau_{0}$ such that, for all $\tau \geq \tau_{0}$

$$
\tau^{\mu}\left|\int_{0}^{T} e^{-\tau t} \varphi(t) d t\right| \geq C .
$$

This together with (2.16) and the trivial estimate

$$
\left|\int_{0}^{T} e^{-\tau t} \varphi(t) d t\right| \leq T^{1 / 2}\|\varphi\|_{L^{2}(0, T)}
$$


yields

$$
\begin{gathered}
C\left|\int_{\partial \Omega} \frac{\partial v}{\partial \nu}\left(R_{\emptyset}(\tau)-R_{D}(\tau)\right) \frac{\partial v}{\partial \nu} d S\right|+O\left(\tau^{\gamma+\mu} e^{-\tau T} e^{2 \sqrt{\tau} h_{\Omega}(\omega)}\right) \\
\leq \tau^{\mu}\left|\int_{\partial \Omega} \int_{0}^{T} e^{-\tau t}\left(v(x) f(x, t ; \tau)-u_{f}(x, t) \frac{\partial v}{\partial \nu}(x)\right) d t d S\right| \\
\leq T^{1 / 2}\|\varphi\|_{L^{2}(0, T)} \tau^{\mu}\left|\int_{\partial \Omega} \frac{\partial v}{\partial \nu}\left(R_{\emptyset}(\tau)-R_{D}(\tau)\right) \frac{\partial v}{\partial \nu} d S\right|+O\left(\tau^{\gamma+\mu} e^{-\tau T} e^{2 \sqrt{\tau} h_{\Omega}(\omega)}\right) .
\end{gathered}
$$

It follows from this and Lemma 2.2 that

$$
\begin{gathered}
C C_{1} \tau^{\mu_{1}-\mu}\left(1+O\left(\tau^{\gamma+\mu-\mu_{1}} e^{-\tau T} e^{2 \sqrt{\tau}\left(h_{\Omega}(\omega)-h_{D}(\omega)\right)}\right)\right) \\
\leq e^{-2 \sqrt{\tau} h_{D}(\omega)}\left|\int_{\partial \Omega} \int_{0}^{T} e^{-\tau t}\left(v(x) f(x, t ; \tau)-u_{f}(x, t) \frac{\partial v}{\partial \nu}(x)\right) d t d S\right| \\
\leq C_{2} T^{1 / 2}\|\varphi\|_{L^{2}(0, T)} \tau^{\mu_{2}}\left(1+O\left(\tau^{\gamma-\mu_{2}} e^{-\tau T} e^{2 \sqrt{\tau}\left(h_{\Omega}(\omega)-h_{D}(\omega)\right)}\right)\right) .
\end{gathered}
$$

Now the formula (1.4) follows from this.

The proof of Lemma 2.2 given below follows the argument in the enclosure method applied to the Helmholtz equation $\left(\triangle+k^{2}\right) u=0[4,5]$. It became much simpler compared with that for the Helmholtz equation since our operator $\triangle-\tau$ is strictly negative for $\tau>0$. Proof of Lemma 2.2. Consider the solution $R(x)$ of the following boundary value problem:

$$
\begin{gathered}
(\triangle-\tau) R=0 \text { in } \Omega \backslash \bar{D}, \\
\frac{\partial R}{\partial \nu}=\frac{\partial v}{\partial \nu} \text { on } \partial D, \\
\frac{\partial R}{\partial \nu}=0 \text { on } \partial \Omega .
\end{gathered}
$$

Multiplying the first equation above by $R$ and integration over $\Omega \backslash \bar{D}$, one gets

$$
\int_{\Omega \backslash \bar{D}}|\nabla R|^{2} d x+\tau \int_{\Omega \backslash \bar{D}}|R|^{2} d x=-\int_{\partial D} \frac{\partial v}{\partial \nu} R d S .
$$

Since the trace operator $H^{1}(\Omega \backslash \bar{D}) \longrightarrow H^{1 / 2}(\partial D)$ is bounded, this right hand side has the bound

$$
\left|\int_{\partial D} \frac{\partial v}{\partial \nu} R d S\right| \leq 2 C\left\|\frac{\partial v}{\partial \nu}\right\|_{H^{-1 / 2}(\partial D)}\|R\|_{H^{1}(\Omega \backslash \bar{D})} .
$$

Now given $\eta>0$ let $\tau$ satisfy $\tau>\eta$. Choose a $\epsilon>0$ in such a way that $\min (1, \eta)>C \epsilon^{2}$. Since we have

$$
2 C\left\|\frac{\partial v}{\partial \nu}\right\|_{H^{-1 / 2}(\partial D)}\|R\|_{H^{1}(\Omega \backslash \bar{D})} \leq C\left(\epsilon^{-2}\left\|\frac{\partial v}{\partial \nu}\right\|_{H^{-1 / 2}(\partial D)}^{2}+\epsilon^{2}\|R\|_{H^{1}(\Omega \backslash \bar{D})}^{2}\right),
$$


it follows from (2.17) and (2.18) that

$$
\|R\|_{H^{1}(\Omega \backslash \bar{D})} \leq\left(\frac{C}{\min (1, \eta)-C \epsilon^{2}}\right)^{1 / 2} \epsilon^{-1}\left\|\frac{\partial v}{\partial \nu}\right\|_{H^{-1 / 2}(\partial D)}
$$

Thus (2.15) implies that

$$
\|R\|_{H^{1}(\Omega \backslash \bar{D})} \leq C^{\prime}\left(\|\nabla v\|_{L^{2}(D)}+\tau\|v\|_{L^{2}(D)}\right)
$$

where $C^{\prime}>0$ is independent of $\tau>\eta$. Noting that $R_{\emptyset}(\tau)(\partial v / \partial \nu)=v, R_{D}(\tau)(\partial v / \partial \nu)=$ $v-R$ on $\partial \Omega$ and the functions $R$ and $v$ are real-valued, we have the integral identity

$$
\begin{gathered}
\int_{\partial \Omega} \frac{\partial v}{\partial \nu}\left(R_{\emptyset}(\tau)-R_{D}(\tau)\right) \frac{\partial v}{\partial \nu} d S \\
=\int_{\Omega \backslash \bar{D}}|\nabla R|^{2} d x+\tau \int_{\Omega \backslash \bar{D}}|R|^{2} d x+\int_{D}|\nabla v|^{2} d x+\tau \int_{D}|v|^{2} d x .
\end{gathered}
$$

A combination of this, (2.19) and the speciality of $v$ gives

$$
2 \tau \int_{D} e^{2 \sqrt{\tau} x \cdot \omega} d x \leq \int_{\partial \Omega} \frac{\partial v}{\partial \nu}\left(R_{\emptyset}(\tau)-R_{D}(\tau)\right) \frac{\partial v}{\partial \nu} d S \leq C_{2} \tau^{3} \int_{D} e^{2 \sqrt{\tau} x \cdot \omega} d x .
$$

Now the conclusion follows from the corresponding estimate for the integral of $e^{2 \sqrt{\tau} x \cdot \omega}$ over $D$ (cf. Propositions 3.1 and 3.2 in [4]).

\section{The use of another $v$}

It is possible to use different $v$ from the exponential solution. In [8] it is shown that: given $p \in \mathbf{R}^{m} \backslash \bar{\Omega}$ one can construct a $v \in H^{2}(\Omega)$ depending on $\tau>0$ and satisfying the equation $(\triangle-\tau) v=0$ in $\bar{\Omega}$ such that

$$
v(x)=e^{-\sqrt{\tau}|x-p|}\left\{|x-p|^{-(m-1) / 2}+O\left(\frac{1}{\sqrt{\tau}}\right)\right\} .
$$

Note that the leading term never vanish on $\bar{\Omega}$; in the case when $m=3$, one can drop the term $O(1 / \sqrt{\tau})$ in the $v$ above.

Using this $v$, we obtain the following formula.

Theorem 3.1. Let $p \in \mathbf{R}^{m} \backslash \bar{\Omega}$ and replace $v$ of $f$ in (1.2) with the $v$ above. Let $u_{f}=u_{f}(x, t)$ be the weak solution of (1.1) for this $f=f(x, t ; \tau)$. Then assuming (1.3), one has the formula

$$
\lim _{\tau \longrightarrow \infty} \frac{1}{2 \sqrt{\tau}} \log \left|\int_{\partial \Omega} \int_{0}^{T} e^{-\tau t}\left(v(x) f(x, t ; \tau)-u_{f}(x, t) \frac{\partial v}{\partial \nu}(x)\right) d t d S\right|=-d_{D}(p),
$$

where $d_{D}(p)$ denotes the distance from $p$ to $D$,

$$
d_{D}(p)=\inf \{|y-p| \mid y \in D\}
$$


Proceeding once more by the method used to prove (1.4), one knows that the key of the proof of Theorem 3.1 is the following lower estimate of the integral of $e^{-2 \sqrt{\tau}|x-p|}$ over $D$.

Proposition 3.2. There exists $\mu \in \mathbf{R}$ such that

$$
\liminf _{\tau \longrightarrow \infty} \tau^{\mu} e^{2 \sqrt{\tau} d_{D}(p)} \int_{D} e^{-2 \sqrt{\tau}|x-p|} d x>0
$$

Proof. Choose $x_{0} \in \partial D$ such that $d_{D}(p)=\left|x_{0}-p\right|$. Since $\partial D$ is smooth, one can find an open ball $B$ with $x_{0} \in \partial B$ and radius $r_{0}$ such that $B \subset D$. Since the integrand is nonnegative, it suffices to prove (3.1) in the case when $D=B$. We observe that

$$
\left|\nabla e^{-2 \sqrt{\tau}|x-p|}\right|=2 \sqrt{\tau} e^{-2 \sqrt{\tau}|x-p|} .
$$

Using the co-area formula (Theorem 2.7.1. on page 76 in [11]), we obtain

$$
\begin{aligned}
\int_{B} e^{-2 \sqrt{\tau}|x-p|} d x & =\frac{1}{2 \sqrt{\tau}} \int_{0}^{\infty} H^{m-1}\left(\left\{x \in B \mid e^{-2 \sqrt{\tau}|x-p|}=t\right\}\right) d t \\
& =\int_{d_{D}(p)}^{\infty} H^{m-1}(S(s)) e^{-2 \sqrt{\tau} s} d s,
\end{aligned}
$$

where $H^{m-1}$ denotes the $m-1$ dimensional Housdorff measure and $S(s)=\{x \in B|| x-$ $p \mid=s\}$.

It is well known that $H^{m-1}$ agrees with the usual definition of $m$-1-dimensional area on an $m$-1-dimensional $C^{1}$ submanifold of $\mathbf{R}^{m}$. This is a corollary of section 3.2 in [2] and consult also p.16 in [11]. Since $S(s)$ for $d_{D}(p)<s<d_{D}(p)+r_{0}$ is a smooth surface $(m=3)$, curve $(m=2), H^{m-1}(S(s))$ coincides with the $m-1$-dimensional area $|S(s)|$. So the problem is reduced to give an estimate for $|S(s)|$ from below as $s \downarrow d_{D}(p)$.

First consider the three-dimensional case. The intersection of the set $\left\{x \in \mathbf{R}^{3}|| x-\right.$ $p \mid=s\}$ with $\partial B$ becomes a circle on a plane and is given by the boundary of an open disc $S^{\prime}(s)$ on the plane. The radius $\delta(s)$ of $S^{\prime}(s)$ is given by the equation

$$
\delta(s)^{2}=r_{0}^{2}-\left\{\frac{r_{0}^{2}+\left(r_{0}+d_{D}(p)\right)^{2}-s^{2}}{2\left(r_{0}+d_{D}(p)\right)}\right\}^{2}
$$

and a simple computation yields $\delta(s)^{2} \geq C\left(s-d_{D}(p)\right)$ as $s \downarrow d_{D}(p)$ for a positive constant $C$. This yields $\left|S^{\prime}(s)\right| \geq \pi C\left(s-d_{D}(p)\right)$ as $s \downarrow d_{D}(p)$. Since $S^{\prime}(s)$ is the projection of $S(s)$ onto the plane mentioned above, we have $|S(s)| \geq\left|S^{\prime}(s)\right|$. Thus we obtain $|S(s)| \geq \pi C\left(s-d_{D}(p)\right)$ as $s \downarrow d_{D}(p)$. From this and (3.2) we obtain the estimate (3.1) with $\mu=1$.

Note that in the two-dimensional case we obtain $|S(s)| \geq 2 C^{1 / 2}\left(s-d_{D}(p)\right)^{1 / 2}$ as $s \downarrow d_{D}(p)$ and (3.1) is valid for $\mu=3 / 4$.

\section{Conclusion and Remarks}

The procedure of extracting the support function of $D$ is extremely simple and summarized as follows. 
(i) Give the direction $\omega \in S^{m-1}$. Fix a large $\tau>0$ and give the heat flux across $\partial \Omega$ over the time interval $] 0, T[$ :

$$
\left.f(x, t ; \tau)=\frac{\partial v}{\partial \nu}(x) \varphi(t),(x, t) \in \partial \Omega \times\right] 0, T[,
$$

where $v(x)=e^{\sqrt{\tau} x \cdot \omega}$ and $\varphi(t)$ satisfies (1.3) for a $\mu$.

(ii) Measure the temperature $u_{f}(x, t)$ on $\partial \Omega$ over the time interval $] 0, T[$.

(iii) Compute the quantity

$$
\frac{1}{2 \sqrt{\tau}} \log \left|\int_{\partial \Omega} \int_{0}^{T} e^{-\tau t}\left(v(x) f(x, t ; \tau)-u_{f}(x, t) \frac{\partial v}{\partial \nu}(x)\right) d t d S\right|
$$

as an approximation of $h_{D}(\omega)$.

As a corollary of Theorem 1.1 we have a constructive proof of the uniqueness of recovering the convex hull of $D$ from infinitely many sets of the temperature and heat flux on $\partial \Omega$ over the time interval ]0, $T$ [. Thus our result can be considered as an extension of the enclosure method of infinitely many measurement version [4].

The method will cover also more general cases without serious difficulty: inclusion, parabolic equations with variable coefficients, Robin boundary condition. And it may be possible to apply our method to the corresponding problems for hyperbolic equations and systems, Stokes system, some governing equations in thermoelasticity, Maxwell systems, etc. Such cases will be reported in detail in forthcoming papers.

Note that in [9] we have already applied the enclosure method of a single measurement version [3] to the problem in three-dimensions. It means that therein the heat flux $f$ is fixed and independent of large parameter $\tau$. The information extracted therein is different from the information obtained in this paper. The analysis developed therein is based on the potential theory and quite delicate. See also [8] for an application of the enclosure method to an inverse source problem for the heat equation in multi-space dimensions.

\section{Acknowledgements}

MI was partially supported by Grant-in-Aid for Scientific Research (C)(No. 18540160) of Japan Society for the Promotion of Science. MK was partially supported by Grantin-Aid for Scientific Research (C)(No. 19540183) of Japan Society for the Promotion of Science.

\section{References}

[1] Dautray, R. and Lions, J-L., Mathematical analysis and numerical methods for sciences and technology, Evolution problems I, Vol. 5, Springer-Verlag, Berlin, 1992.

[2] Federer, H., Geometric measure theory, Springer-Verlag, New York, Heidelberg, 1969.

[3] Ikehata, M., Enclosing a polygonal cavity in a two-dimensional bounded domain from Cauchy data, Inverse Problems, 15(1999), 1231-1241. 
[4] Ikehata, M., Reconstruction of the support function for inclusion from boundary measurements, J. Inv. Ill-Posed Problems, 8(2000), 367-378.

[5] Ikehata, M., How to draw a picture of an unknown inclusion from boundary measurements. Two mathematical inversion algorithms, J. Inv. Ill-Posed Problems, 7(1999), $255-271$.

[6] Ikehata, M., Extracting discontinuity in a heat conductive body. One-space dimensional case, Applicable Analysis, 86(2007), no. 8, 963-1005.

[7] Ikehata, M., Virtual signal in the heat equation and the enclosure method, Inverse Problems in Applied Sciences-towards breakthrough-, Journal of Physics: Conference Series 73 (2007)012010.

[8] Ikehata, M., An inverse source problem for the heat equation and the enclosure method, Inverse Problems, 23(2007), 183-202.

[9] Ikehata, M. and Kawashita, M., An inverse problem for a three-dimensional heat equation in thermal imaging and the enclosure method, preprint.

[10] Vessella, S., Quantitative estimates of unique continuation for parabolic equations, determination of unknown time-varying boundaries and optimal stability estimates, Topical Review, Inverse Problems, 24(2008)023001(81pp).

[11] Ziemer, W.P., Weakly differentiable functions, Graduate texts in mathematics, 120, Springer-Verlag, New York, 1989.

e-mail address

ikehata@math.sci.gunma-u.ac.jp

kawasita@math.sci.hiroshima-u.ac.jp 